\title{
Analyzing linear systems containing strict inequalities via evenly convex hulls ${ }^{1}$
}

\author{
Miguel A. Goberna and Margarita M. L. Rodríguez \\ Department of Statistics and Operations Research \\ Faculty of Sciences \\ University of Alicante \\ E-03080 Alicante, Spain. \\ e-mail:mgoberna@ua.es,marga.rodriguez@ua.es
}

\begin{abstract}
The evenly convex hull of a given set is the intersection of all the open halfspaces which contain such set (hence the convex hull is contained in the evenly convex hull). This paper deals with finite dimensional linear systems containing strict inequalities and (possibly) weak inequalities as well as equalities. The number of inequalities and equalities in these systems is arbitrary (possibly infinite). For such kind of systems a consistency theorem is provided and those strict inequalities (weak inequalities, equalities) which are satisfied for every solution of a given system are characterized. Such results are formulated in terms of the evenly convex hull of certain sets which depend on the coefficients of the system.
\end{abstract}

AMS classification (2000): 15A39; 90C34; 52A40.

Keywords: convex programming; linear programming; evenly convex hull; existence theorems, consequence relations.

\footnotetext{
${ }^{1}$ This work was supported by the MCYT of Spain and FEDER of UE, Grant BFM2002-04114-C0201.
} 


\section{Introduction}

This paper deals with linear inequality systems in $\mathbb{R}^{n}$ of the form

$$
\sigma=\left\{a_{t}^{\prime} x>b_{t}, t \in S ; a_{t}^{\prime} x \geq b_{t}, t \in W ; a_{t}^{\prime} x=b_{t}, t \in E\right\}
$$

with $S \neq \emptyset, S, W$ and $E$ pairwise disjoint sets, $a_{t} \in \mathbb{R}^{n}$ and $b_{t} \in \mathbb{R}$ for all $t \in T:=S \cup W \cup E$ (a possibly infinite set). We denote $V=S \cup W$. The main purpose of the paper is to characterize those systems $\sigma$ for which there exist solutions (i.e., the class of consistent systems). The secondary purpose consists of characterizing those (weak and strict) inequalities and equalities which are satisfied by every solution of $\sigma$. In other words, this problem consists of characterizing the consequence inequalities or equalities of a given consistent system.

Systems as $\sigma$ with $|T|<\infty$ are considered in [18] and [29], where the concept of legal linear combination is defined. A basic result in [29], the so-called Kuhn-Fourier Theorem, characterizes the consistency of these systems in terms of legal linear combinations. Other existence theorems for particular families of finite systems, formulated as alternative theorems, have been collected in [19]. We summarize in Table 1 the information on the most representative results spread on 11 references chronologically ordered.

\begin{tabular}{|c|c|c|c|c|c|c|}
\hline Ref. & Year & $S$ & $W$ & $E$ & $b$. & Cond. \\
\hline [13] & 1873 & fin. & $\emptyset$ & $\phi$ & 0 & Not \\
\hline [4] & 1921-22 & fin. & $\emptyset$ & $\emptyset$ & arb. & Not \\
\hline [25] & 1936 & fin. & fin. & fin. & 0 & Not \\
\hline$[8]$ & 1960 & $\emptyset$ & fin. & $\emptyset$ & arb. & Not \\
\hline [31] & 1966 & Q & arb & a & arh & Not \\
\hline$[6]$ & 1968 & $\varphi$ & ard. & $\varphi$ & aro. & Nol \\
\hline [29] & 1970 & fin. & fin. & fin. & arb. & Not \\
\hline [12] & 1984 & arb. & arb. & arb. & 0 & Yes \\
\hline [12] & 1984 & arb. & $\phi$ & $\emptyset$ & 0 & Yes \\
\hline [30] & 1999 & fin. & fin. & fin. & arb. & Not \\
\hline [10] & 2003 & arb. & arb. & $\emptyset$ & arb. & Yes \\
\hline
\end{tabular}

The columns 3, 4 and 5 in Table 1 inform on the cardinality of the index sets, which can be empty, finite or arbitrary (abbreviated as " $\emptyset "$, "fin." and "arb.", respectively). Column 6 informs about the kind of right-hand side scalars $b_{t}$ the theorems deal with $(0$, in the case of homogeneous systems, and arbitrary, otherwise). Finally, column 7 informs about the full generality or not of the corresponding existence theorem. Observe that all the known existence theorems for systems containing an arbitrary number of strict inequalities are only 
valid provided that a suitable closedness assumption holds.

In [30], classical alternative theorems concerning the consistency of finite linear inequality systems are reformulated in a common, general form. In such systems, matricial notation is used and inequalities of the form $A x \geq b$ mean $A x \geqq b$ but $A x \neq b$. Such inequalities are equivalent to $A x \geqq b$ and $e^{\prime} A x>e^{\prime} b$, with $e^{\prime}=(1, \ldots, 1)$ of appropriate size, so that these systems are particular cases of (1.1) and all the results in [30] can be obtain as particular cases of our Theorem 3.1.

[10, Proposition 2.1] provides a sufficient condition as well as a necessary condition for $\sigma$ to be consistent when $E=\emptyset$, but there is a gap between both conditions, i.e., there exists a nonempty class of system which cannot be classified as either consistent or inconsistent. Theorem 3.1 shows that, as Dr. Martínez-Legaz conjectured, the use of evenly convex sets allows the elimination of the mentioned gap.

A set is evenly convex if it is the intersection of some family (possibly empty) of open halfspaces. This class of sets was introduced by Fenchel [7] in order to extend the polarity theory to nonclosed convex sets. Later, they have been applied in quasiconvex programming [22, 23, 26 and 27], mathematical economy [24] and linear inequality systems [10] (as far as the evenly convex sets are the solution sets of linear systems of either weak or strict inequalities). New characterizations of this class of sets have been given recently in [5].

The evenly convex hull of a set $X$ is the smallest evenly convex set which contains $X$. Section 2 characterizes the evenly convex hull of a set and analyzes those properties which could help in its effective calculus. Section 3 deals with the existence of solutions. The main result there is the existence Theorem 3.1, which characterizes the consistent systems containing strict inequalities without any assumption. Finally, Section 4 characterizes the weak (strict) inequalities and the equalities which are consequence of a given consistent system.

Farkas' Lemma provides a dual characterization of the containment of a polyhedral convex set in a closed halfspace. This famous result has been extended in different directions (see the survey in [15]):

1. Eliminating the finiteness assumptions on the number of constraints or the space dimension. Thus, the classical paper [14] deals with Euclidean spaces, [3] with real locally convex spaces, [6] with normed spaces, [31] with vector topological spaces, and [1] with matrix spaces.

2. Replacing polyhedral convex sets by closed convex sets and/or closed halfspaces by a different class of sets. Thus, [21] characterizes the containment of polyhedral sets in polyhedral 
sets as well as in nonconvex sets determined by finitely many quadratic constraints whereas [16] replaces polyhedral convex sets by the solution sets of convex semi-infinite systems and eliminates the finiteness assumption on the number of quadratic constraints.

These dual characterizations of containments have important applications in mathematical programming (duality, Lagrange multipliers, and minimax theory) and data mining (see, e.g., [20], [2], and [17]). These are the potential application fields of the extensions provided in Section 4 of this paper, where closed convex sets are replaced by evenly convex sets (class which also includes relatively open convex sets) and closed halfspaces by arbitrary halfspaces.

Concerning mathematical programming, we have shown in [10] several applications of the existence question posed on systems such that $S \neq \emptyset$ and $W=E=\emptyset$. Let us observe now that the typical stability condition for a linear semi-infinite program in $\mathbb{R}^{n}$

$$
\operatorname{Min} c^{\prime} x \quad \text { s.t. } \quad a_{t}^{\prime} x \geq b_{t}, t \in W
$$

is the existence of a strong Slater element (see, e.g., [11]), i.e., the consistency of

$$
\left\{x_{n+1}>0 ; a_{t}^{\prime} x-x_{n+1} \geq b_{t}, t \in W\right\},
$$

so that $|S|=1$ and $W$ is infinite, i.e., mixed systems arise in a natural way.

Similarly, the stability condition for a linear program

$$
\begin{array}{ll}
\text { Min } & c^{\prime} x \\
\text { s.t. } & a_{t}^{\prime} x \geq b_{t}, t \in W \\
& a_{t}^{\prime} x=b_{t}, t \in E
\end{array}
$$

is the linear independence of $\left\{a_{t}, t \in E\right\}$ (if $E \neq \emptyset$ ) together with the consistency of $\left\{a_{t}^{\prime} x>b_{t}, t \in W ; a_{t}^{\prime} x=b_{t}, t \in E\right\}$ ([9, Theorem 4.6]).

Now let us introduce the necessary notation. Given a nonempty set $X \subset \mathbb{R}^{p}, p \in \mathbb{N}$, we denote by $\operatorname{cl} X$, conv $X$, cone $X$, eco $X$ and $\operatorname{span} X$ the closure of $X$, the convex hull of $X$, the convex cone generated by $X$, the evenly convex hull of $X$ and the linear subspace of $\mathbb{R}^{n}$ spanned by $X$, respectively. Moreover, we define cone $\emptyset=\operatorname{span} \emptyset=\left\{0_{p}\right\}$. Some of the above sets can be easily described by means of the space of generalized finite sequences, $\mathbb{R}^{(T)}$, whose elements are the functions $\lambda: T \rightarrow \mathbb{R}$ such that $\lambda_{t} \neq 0$ only on a finite subset of $T$. The convex cone, in $\mathbb{R}^{(T)}$, of the nonnegative finite sequences is $\mathbb{R}_{+}^{(T)}$. If $X \neq \emptyset$ is convex, we denote by $\operatorname{dim} X$ the dimension of $X$. Given two sets $M \subset \mathbb{R}$ and $X \subset \mathbb{R}^{n}$, we denote $M X=\{\mu x \mid \mu \in M, x \in X\}$ if $M \neq \emptyset \neq X$ and $\left\{0_{n}\right\}$ otherwise. Finally, given 
a map $A: R^{p} \rightarrow R^{q}, X \subset R^{p}$ and $Y \subset R^{q}$, we denote $A X=\{A(x) \mid x \in X\}$ and $A^{-1} Y=\left\{x \in \mathbb{R}^{p} \mid A(x) \in Y\right\}$.

If $S=\emptyset$ (case not considered here), the following results are straightforward consequence of [6, Theorem 1] and [31, Theorem 2], respectively.

Proposition 1.1. A system $\left\{a_{t}^{\prime} x \geq b_{t}, t \in W ; a_{t}^{\prime} x=b_{t}, t \in E\right\}$ is consistent if and only if

$$
\left(\begin{array}{c}
0_{n} \\
1
\end{array}\right) \notin \operatorname{cl} \text { cone }\left\{\left(\begin{array}{l}
a_{t} \\
b_{t}
\end{array}\right), t \in W ; \pm\left(\begin{array}{l}
a_{t} \\
b_{t}
\end{array}\right), t \in E\right\} .
$$

Proposition 1.2. A weak inequality $a^{\prime} x \geq b$ is a consequence of a consistent system $\left\{a_{t}^{\prime} x \geq b_{t}, t \in W ; a_{t}^{\prime} x=b_{t}, t \in E\right\}$ if and only if $\left(\begin{array}{l}a \\ b\end{array}\right) \in \mathrm{cl} K$, where

$$
K=\text { cone }\left\{\left(\begin{array}{c}
a_{t} \\
b_{t}
\end{array}\right), t \in W ; \pm\left(\begin{array}{c}
a_{t} \\
b_{t}
\end{array}\right), t \in E ;\left(\begin{array}{c}
0_{n} \\
-1
\end{array}\right)\right\}
$$

Consequently, a strict inequality $a^{\prime} x>b$ is a consequence of a consistent system $\left\{a_{t}^{\prime} x \geq b_{t}, t \in W ; a_{t}^{\prime} x=b_{t}, t \in E\right\}$ if and only if

$$
\left(\begin{array}{l}
a \\
b
\end{array}\right) \in \operatorname{cl} K \text { and }\left(\begin{array}{c}
0_{n} \\
1
\end{array}\right) \in \operatorname{cl}\left[K+\operatorname{span}\left\{\left(\begin{array}{l}
a \\
b
\end{array}\right)\right\}\right] \text {. }
$$

We shall use the following characterizations of the evenly convex sets.

Proposition 1.3 ([10]). Given $C \subset \mathbb{R}^{n}$ such that $\emptyset \neq C \neq \mathbb{R}^{n}$, the following conditions are equivalent to each other:

(i) $C$ is evenly convex;

(ii) $C$ is a convex set and for each $x \in \mathbb{R}^{n} \backslash C$ there exists a hyperplane $H$ such that $x \in H$ and $H \cap C=\emptyset$; and

(iii) $C$ is the result of eliminating from a closed convex set the union of some family of its exposed faces.

\section{The evenly convex hull}

If conv $X \varsubsetneqq \mathbb{R}^{n}$, the intersection of all the open halfspaces containing $X$ is the minimal evenly convex set which contains $X$, i.e., it is eco $X$. Alternatively, if conv $X=\mathbb{R}^{n}$ (i.e., if there does not exist a halfspace containing $X$ ), then eco $X=\mathbb{R}^{n}$. Obviously, $X$ is evenly convex if and only if eco $X=X$. This happens, for instance, if $X$ is either a closed or a relatively 
open convex set. Consequently, if $X$ is a compact (open) set, then conv $X$ is a compact (open) convex set and eco $X=\operatorname{conv} X$. This is the case, in particular, if $|X|<\infty$. From the definition of evenly convex set, given $\bar{x} \in \mathbb{R}^{n}, \bar{x} \notin$ eco $X$ if and only if there exists $z \in \mathbb{R}^{n}$ such that $(x-\bar{x})^{\prime} z>0$, for all $x \in X$. In particular,

$$
0_{n} \notin \operatorname{eco} X \text { if and only if }\left\{x^{\prime} z>0, x \in X\right\} \text { is consistent. }
$$

(2.1) can be interpreted as a counterpart of the Farkas' Lemma for homogeneous systems of strict linear inequalities.

It can be easily seen that, for any $X \subset \mathbb{R}^{n}$,

$$
\text { eco conv } X=\operatorname{eco} X=\operatorname{conv} \text { eco } X \text {. }
$$

Proposition 2.1. For any $X \subset \mathbb{R}^{n}$, eco $X$ is the result of eliminating from cl conv $X$ the union of all its exposed faces which do not intersect $X$. So $\operatorname{dim} \operatorname{eco} X=\operatorname{dim} \operatorname{conv} X$ and eco cone $X=\operatorname{cl}$ cone $X$.

Proof. Let $C$ be the result of eliminating from $\operatorname{cl} \operatorname{conv} X$ the union of all its exposed faces which do not intersect $X$. According to Proposition 1.3, $C$ is evenly convex, so that eco $X \subset C$. Assume that the inclusion is strict and let $z \in C \backslash$ (eco $X)$. Since eco $X$ is evenly convex, by Proposition 1.3(ii), there exists $c \in \mathbb{R}^{n} \backslash\left\{0_{n}\right\}$ and $\alpha \in \mathbb{R}$ such that $c^{\prime} x<\alpha$ for all $x \in$ eco $X$ and $c^{\prime} z=\alpha$. Then $z \in Y:=\left\{y \in \operatorname{cl} \operatorname{conv} X \mid c^{\prime} y=\alpha\right\}$ and, since

$$
\operatorname{conv} X \subset \operatorname{eco} X \subset \operatorname{cl} \operatorname{conv} X
$$

$Y$ is an exposed face of $\operatorname{cl} \operatorname{conv} X$ which does not intersect $X$. This contradicts $z \in C$.

The dimensional equation is a consequence of (2.3). Finally, observe that any supporting hyperplane of clcone $X$ contains $0_{n}$ so that any exposed face intersects cone $X$ and eco cone $X=\operatorname{cl}$ cone $X$.

Observe that, for any $X \subset \mathbb{R}^{n}$,

$$
\text { cone eco } X \subset \text { eco cone } X=\operatorname{cl} \text { cone } X \text {. }
$$

and the inclusion can be strict. 
Example 2.1. Given the set $X=\left\{x \in \mathbb{R}^{2} \mid x_{2}=\frac{1}{1+x_{1}^{2}}\right\}$, we have

$$
\operatorname{cl}(\operatorname{conv} X)=\mathbb{R} \times[0,1]
$$

and

$$
\text { eco } X=\mathbb{R} \times] 0,1] \text {. }
$$

We can observe that eco $X$ is the result of eliminating from $\operatorname{cl}(\operatorname{conv} X)$ the only exposed face which does not intersect $X$ (the line $R \times\{0\}$ ).

On the other hand, cone eco $X=$ cone $X=(\mathbb{R} \times] 0,+\infty[) \cup\left\{0_{2}\right\}$ whereas eco cone $X=$ cl cone $X=R \times[0,+\infty[$, so that the inclusion in (2.4) is strict.

Lemma 2.1. Let $A, B, C$ be nonempty sets in $\mathbb{R}^{n}$. Then the following statements hold:

(i) conv $A+\operatorname{cone} B=\operatorname{conv}\left(A+\mathbb{R}_{+} B\right)$.

(ii) $\operatorname{conv} A+\operatorname{cone} B+\operatorname{span} C=\operatorname{conv}\left(A+\mathbb{R}_{+} B+\mathbb{R} C\right)$.

Proof. (i) If $z \in \operatorname{conv} A+$ cone $B$, then there exist $\left\{a_{1}, \ldots, a_{m}\right\} \subset A,\left\{b_{1}, \ldots, b_{p}\right\} \subset B$ and nonnegative scalars $\left\{\lambda_{1}, \ldots, \lambda_{m}\right\}$ and $\left\{\mu_{1}, \ldots, \mu_{p}\right\}$, with $\sum_{i=1}^{m} \lambda_{i}=1$, such that

$$
z=\sum_{i=1}^{m} \lambda_{i} a_{i}+\sum_{j=1}^{p} \mu_{j} b_{j}
$$

We can assume $\lambda_{i}>0$ for all $i=1, \ldots, m$. If $\mu_{j}=0$ for all $j=1, \ldots, p$, then $z \in \operatorname{conv} A \subset \operatorname{conv}\left(A+\mathbb{R}_{+} B\right)$. Otherwise, we can assume $\sum_{j=1}^{p} \mu_{j}>0$ and we can write

$$
z=\sum_{i=1}^{m-1} \lambda_{i} a_{i}+\sum_{j=1}^{p}\left(\frac{\lambda_{m} \mu_{j}}{\sum_{j=1}^{p} \mu_{j}}\right)\left[a_{m}+\left(\frac{\sum_{j=1}^{p} \mu_{j}}{\lambda_{m}}\right) b_{j}\right] .
$$

Since $a_{i} \in A+\{0\} B \subset A+\mathbb{R}_{+} B, i=1, \ldots, m-1, a_{m}+\left(\frac{\sum_{j=1}^{p} \mu_{j}}{\lambda_{m}}\right) b_{j} \in A+\mathbb{R}_{+} B$, $j=1, \ldots, p$, and $\sum_{i=1}^{m-1} \lambda_{i}+\sum_{j=1}^{p}\left(\frac{\lambda_{m} \mu_{j}}{\sum_{j=1}^{p} \mu_{j}}\right)=1$, we have $z \in \operatorname{conv}\left(A+\mathbb{R}_{+} B\right)$.

Hence conv $A+\operatorname{cone} B \subset \operatorname{conv}\left(A+\mathbb{R}_{+} B\right)$. 
Conversely, any $z \in \operatorname{conv}\left(A+\mathbb{R}_{+} B\right)$ can be written as

$$
z=\sum_{i=1}^{m} \lambda_{i}\left(a_{i}+\mu_{i} b_{i}\right)
$$

with $a_{i} \in A, b_{i} \in B, \lambda_{i} \geq 0$ and $\mu_{i} \geq 0, i=1, \ldots, m$, and $\sum_{i=1}^{m} \lambda_{i}=1$.

Then

(ii) By statement (i),

$$
z=\sum_{i=1}^{m} \lambda_{i} a_{i}+\sum_{i=1}^{m}\left(\lambda_{i} \mu_{i}\right) b_{i} \in \operatorname{conv} A+\operatorname{cone} B .
$$

$$
\begin{aligned}
& \operatorname{conv} A+\operatorname{cone} B+\operatorname{span} C=\operatorname{conv} A+\operatorname{cone}[B \cup C \cup(-C)] \\
& =\operatorname{conv}\left\{A+\mathbb{R}_{+}[B \cup C \cup(-C)]\right\} \subset \operatorname{conv}\left(A+\mathbb{R}_{+} B+\mathbb{R} C\right) .
\end{aligned}
$$

The reverse inclusion can be proved as in (i).

Proposition 2.2. Let $A, B, C$ be nonempty sets in $\mathbb{R}^{n}$. Then the following statements hold:

(i) eco $(\operatorname{conv} A+\operatorname{cone} B)=\operatorname{eco}\left(A+\mathbb{R}_{+} B\right)$.

(ii) eco $(\operatorname{conv} A+\operatorname{cone} B+\operatorname{span} C)=\operatorname{eco}\left(A+\mathbb{R}_{+} B+\mathbb{R} C\right)$.

Proof. (i) According to Lemma 2.1, part (i), and applying (2.2), we have

$$
\operatorname{eco}(\operatorname{conv} A+\operatorname{cone} B)=\operatorname{eco} \operatorname{conv}\left(A+\mathbb{R}_{+} B\right)=\operatorname{eco}\left(A+\mathbb{R}_{+} B\right) .
$$

(ii) Combining part (ii) of Lemma 2.1 and (2.2), we get

$\operatorname{eco}(\operatorname{conv} A+\operatorname{cone} B+\operatorname{span} C)=\operatorname{ecoconv}\left(A+\mathbb{R}_{+} B+\mathbb{R} C\right)=\operatorname{eco}\left(A+\mathbb{R}_{+} B+\mathbb{R} C\right)$.

Proposition 2.3. Given two sets $X \subset \mathbb{R}^{n}$ and $Y \subset \mathbb{R}^{m}$,

$$
\operatorname{eco}(X \times Y)=(\operatorname{eco} X) \times(\operatorname{eco} Y)
$$

Proof. Since the product of evenly convex sets is evenly convex too [10, Proposition 3.6], $\operatorname{eco}(X \times Y) \subset(\operatorname{eco} X) \times($ eco $Y)$.

Now we assume $(\bar{x}, \bar{y}) \notin$ eco $(X \times Y)$. Let $(c, d) \in \mathbb{R}^{n+m} \backslash\left\{0_{n+m}\right\}$ and $\alpha \in \mathbb{R}$ such that $c^{\prime} x+d^{\prime} y>\alpha$ for all $(x, y) \in X \times Y$ and

$$
c^{\prime} \bar{x}+d^{\prime} \bar{y} \leq \alpha
$$


(or even $c^{\prime} \bar{x}+d^{\prime} \bar{y}=\alpha$ ). We have either $c \neq 0_{n}$ or $d \neq 0_{m}$ (maybe both).

If $c \neq 0_{n}$ and $d=0_{m}$, then $c^{\prime} x>\alpha$ for all $x \in X$ and $c^{\prime} \bar{x} \leq \alpha$, so that $\bar{x} \notin$ eco $X$ and $(\bar{x}, \bar{y}) \notin \operatorname{eco} X \times \operatorname{eco} Y$. We get the same conclusion if $c=0_{n}$ and $d \neq 0_{m}$.

Then, we can assume $c \neq 0_{n}$ and $d \neq 0_{m}$. For each $y \in Y$, the set $\left\{x \in \mathbb{R}^{n} \mid c^{\prime} x>\alpha-d^{\prime} y\right\}$ is an open halfspace containing $X$, so that, if $\bar{x} \in \operatorname{eco} X$, we have $c^{\prime} \bar{x}>\alpha-d^{\prime} y$. Combining this expression and (2.5), we obtain $d^{\prime} y>d^{\prime} \bar{y}$ for all $y \in Y$, so that $\bar{y} \notin \operatorname{eco} Y$ and $(\bar{x}, \bar{y}) \notin \operatorname{eco} X \times \operatorname{eco} Y$.

So $(\bar{x}, \bar{y}) \notin \operatorname{eco} X \times \operatorname{eco} Y$ in either case and we can conclude $(\operatorname{eco} X) \times(\operatorname{eco} Y) \subset$ eco $(X \times Y)$.

Example 2.2. Let $A: \mathbb{R}^{2 n} \rightarrow \mathbb{R}^{n}$ such that $A(x, z)=x+z$ and let $X=C_{1} \times$ $C_{2}$, where $C_{1}=\left\{x \in \mathbb{R}^{2} \mid t x_{1}+(1-t) x_{2} \geq t-t^{2}, t \in[0,1], x_{1}+x_{2} \leq 1\right\}$ and $C_{2}=$ $\left\{x \in \mathbb{R}^{2} \mid x_{1} \geq 0 ; x_{2} \geq 0 ; x_{1}+x_{2}>0\right\}$. It can be shown that we have eco $X=X$ by Proposition 2.3 and so

$$
A(\operatorname{eco} X) \varsubsetneqq \operatorname{eco} A X
$$

(see Figures 3-5 in [10]).

The next result shows that the corresponding inclusion always holds.

Proposition 2.4. Let $X \neq \emptyset$ be a set in $\mathbb{R}^{m}$ and let $A: \mathbb{R}^{m} \rightarrow \mathbb{R}^{n}$ be a linear transformation. Then $A(\operatorname{eco} X) \subset \operatorname{eco} A X$.

Proof. Let $\bar{x} \in \operatorname{eco} X$ and assume $A \bar{x} \notin$ eco $A X$. Then, there exist $c \in \mathbb{R}^{n} \backslash\left\{0_{n}\right\}$ and $\alpha \in \mathbb{R}$ such that $c^{\prime} A x>\alpha$ for all $x \in X$ and $c^{\prime} A \bar{x} \leq \alpha$.

Taking $d:=c^{\prime} A \in \mathbb{R}^{m}$, we have $d^{\prime} x>\alpha$ for all $x \in X$ and $d^{\prime} \bar{x} \leq \alpha$, with $d \neq 0_{m}$ (otherwise $0 \leq \alpha<0$ ), and this contradicts $\bar{x} \in \operatorname{eco} X$.

Corollary 2.1. Given two nonempty sets $X, Y \subset \mathbb{R}^{n}$,

$$
\operatorname{eco} X+\operatorname{eco} Y \subset \operatorname{eco}(X+Y) \text {. }
$$

Proof. Let $A: \mathbb{R}^{2 n} \rightarrow \mathbb{R}^{n}$ be the linear transformation defined as $A(x, y)=x+y, x, y \in \mathbb{R}^{n}$. Then, applying Propositions 2.3, we have

$$
\operatorname{eco} X+\operatorname{eco} Y=A[(\operatorname{eco} X) \times(\operatorname{eco} Y)]=A[\operatorname{eco}(X \times Y)]
$$


and, by Proposition 2.4,

$$
A[\operatorname{eco}(X \times Y)] \subset \operatorname{eco}[A(X \times Y)]=\operatorname{eco}(X+Y)
$$

Combining (2.7) and (2.8), we obtain (2.6).

Since the sum of evenly convex sets is not necessarily evenly convex (recall Example 2.2), the inclusion (2.6) cannot be replaced by an equality.

Proposition 2.5. Let $X$ be a nonempty set in $\mathbb{R}^{n}$ and let $A: \mathbb{R}^{m} \rightarrow \mathbb{R}^{n}$ be a linear transformation such that $A^{-1} X \neq \emptyset$. Then

$$
\operatorname{eco}\left(A^{-1} X\right) \subset A^{-1}(\text { eco } X)
$$

Proof. According to [10, Proposition 3.5], $A^{-1}$ (eco $X$ ) is an evenly convex set which contains $A^{-1} X$, so that (2.9) holds.

The reverse inclusion is not true.

Example 2.3. Let $X=(] 0,+\infty[\times] 0,+\infty[) \cup\left\{0_{2}\right\} \subset \mathbb{R}^{2}$ and let $A: \mathbb{R}^{2} \rightarrow \mathbb{R}^{2}$ be the linear transformation defined as $A\left(x_{1}, x_{2}\right)=\left(x_{1}, 0\right)$.

$A^{-1} X=\{0\} \times \mathbb{R}$ is an evenly convex set whereas $A^{-1}($ eco $X)=\mathbb{R}_{+} \times \mathbb{R}$. So that, eco $\left(A^{-1} X\right)=A^{-1} X \varsubsetneqq A^{-1}($ eco $X)$.

Since the intersection of evenly convex sets is evenly convex too, we get the next result.

Proposition 2.6. Given a family of nonempty sets in $\mathbb{R}^{n},\left\{X_{i} \mid i \in I\right\}$,

$$
\operatorname{eco}\left(\bigcap_{i \in I} X_{i}\right) \subset \bigcap_{i \in I}\left(\operatorname{eco} X_{i}\right)
$$

Proof. The proof is trivial.

The next example shows that the inclusion in (2.10) cannot be replaced by an equality (even though $|I|<\infty)$.

Example 2.4. Let $X_{1}=\mathbb{R}^{2} \times\{0\}$ and

$$
X_{2}=\operatorname{conv}\left[\left\{\left(\begin{array}{c}
-\cos t \\
-\sin t \\
-1
\end{array}\right), t \in\right] 0,2 \pi[\}+\mathbb{R}_{+}\left\{\left(\begin{array}{l}
1 \\
0 \\
1
\end{array}\right)\right\}\right] \cup\left\{\left(\begin{array}{l}
1 \\
0 \\
1
\end{array}\right)\right\}
$$




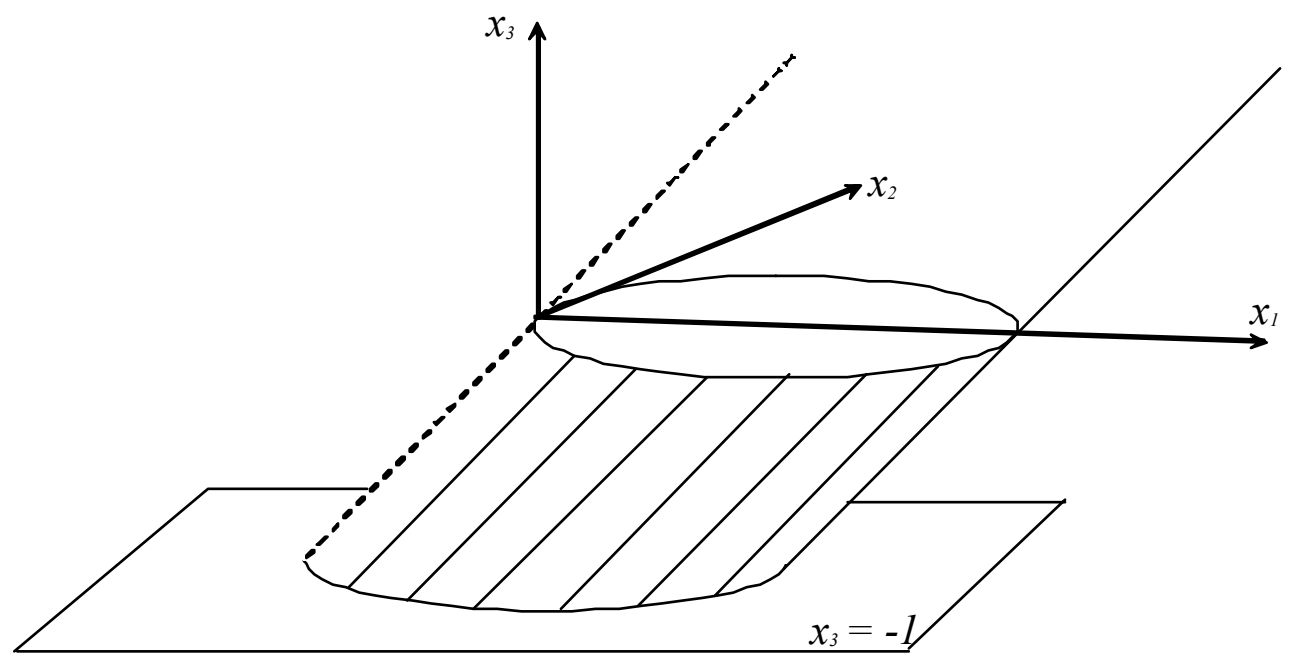

Figure 2.1

(see Figure 2.1).

Since eco $X_{1}=X_{1}$ and eco $X_{2}=\operatorname{cl} X_{2}$, we have $\left(\operatorname{eco} X_{1}\right) \cap\left(\operatorname{eco} X_{2}\right)=$ $\left\{\left(x_{1}, x_{2}, 0\right) \in \mathbb{R}^{3} \mid\left(x_{1}-1\right)^{2}+x_{2}^{2} \leq 1\right\}$ whereas

$$
\operatorname{eco}\left(X_{1} \cap X_{2}\right)=X_{1} \cap X_{2}=\left\{\left(x_{1}, x_{2}, 0\right) \in \mathbb{R}^{3} \mid\left(x_{1}-1\right)^{2}+x_{2}^{2} \leq 1\right\} \backslash\left\{0_{3}\right\}
$$

Proposition 2.7. Given a nonempty bounded set $X \subset \mathbb{R}^{n}$,

$$
\operatorname{cl}(\operatorname{eco} X)=\operatorname{eco}(\operatorname{cl} X)=\operatorname{conv}(\operatorname{cl} X)
$$

Proof. By [28, Theorem 17.2],

$$
\operatorname{cl}(\operatorname{conv} X)=\operatorname{conv}(\operatorname{cl} X)
$$

and, since $\operatorname{cl} X$ is compact, we have

$$
\operatorname{eco}(\operatorname{cl} X)=\operatorname{conv}(\operatorname{cl} X)
$$

Combining (2.11) and (2.12), we obtain

$$
\operatorname{eco}(\operatorname{cl} X)=\operatorname{cl}(\operatorname{conv} X)
$$


so that eco $(\operatorname{cl} X)$ is a closed set containing eco $X$ and

$$
\operatorname{cl}(\operatorname{eco} X) \subset \operatorname{eco}(\operatorname{cl} X)
$$

We obtain the opposite inclusion observing that $\operatorname{cl}(\operatorname{eco} X)$ is an evenly convex set containing $\mathrm{cl} X$.

The boundedness condition in the last result is not superfluous.

Example 2.1 (revisited). For the unbounded set $X$, we have

$$
\operatorname{eco}(\operatorname{cl} X)=\operatorname{eco} X=\mathbb{R} \times] 0,1] \neq \mathbb{R} \times[0,1]=\operatorname{cl}(\operatorname{eco} X) .
$$

\section{Existence of solutions}

The main result in this paper characterizes the consistency of linear systems with strict inequalities in terms of the evenly convex hull of a certain set depending on the coefficients.

For the sake of brevity in the proofs, given a subset of indices $I \subset T$, we denote by $C_{I}$ the $\operatorname{set}\left\{\left(\begin{array}{c}a_{t} \\ b_{t}\end{array}\right), t \in I\right\}$.

Lemma 3.1. The following conditions are equivalent to each other:

(i) $\left\{a_{t}^{\prime} x>b_{t}, t \in S\right\}$, with $S \neq \emptyset$, is consistent;

(ii) $0_{n+1} \notin$ eco $\left[\left\{\left(\begin{array}{l}a_{t} \\ b_{t}\end{array}\right), t \in S\right.\right.$; $\left.\left.\left(\begin{array}{c}0_{n} \\ -1\end{array}\right)\right\}\right]$;

(iii) $0_{n+1} \notin$ eco $\left[\left\{\left(\begin{array}{c}a_{t} \\ b_{t}\end{array}\right), t \in S\right\}\right]$ and $\left(\begin{array}{c}0_{n} \\ 1\end{array}\right) \notin \operatorname{clcone}\left\{\left(\begin{array}{c}a_{t} \\ b_{t}\end{array}\right), t \in S\right\}$.

Proof. (i) $\Rightarrow$ (ii) Let $x^{1} \in \mathbb{R}^{n}$ be a solution of $\left\{a_{t}^{\prime} x>b_{t}, t \in S\right\}$. Then $\left(\begin{array}{c}x^{1} \\ -1\end{array}\right) \in \mathbb{R}^{n+1}$ is a solution of the homogeneous system

$$
\left\{\left(\begin{array}{l}
a_{t} \\
b_{t}
\end{array}\right)^{\prime}\left(\begin{array}{c}
x \\
x_{n+1}
\end{array}\right)>0, t \in S ;\left(\begin{array}{c}
0_{n} \\
-1
\end{array}\right)^{\prime}\left(\begin{array}{c}
x \\
x_{n+1}
\end{array}\right)>0\right\},
$$

whose consistency is equivalent to condition (ii) by (2.1).

(ii) $\Rightarrow$ (iii) Since eco $C_{S} \subset$ eco $\left[C_{S} \cup\left\{\left(\begin{array}{c}0_{n} \\ -1\end{array}\right)\right\}\right]$, condition (ii) entails $0_{n+1} \notin$ eco $C_{S}$.

On the other hand, condition (ii) implies that there exists a vector $c \in \mathbb{R}^{n+1}$ such that $c^{\prime} z>0$ for all $z \in C_{S}$ and $c^{\prime}\left(\begin{array}{c}0_{n} \\ -1\end{array}\right)>0$. Hence, $X=:\left\{x \in \mathbb{R}^{n+1} \mid c^{\prime} x \geq 0\right\}$ is an homogeneous closed 
halfspace containing $C_{S}$ such that $\left(\begin{array}{c}0_{n} \\ 1\end{array}\right) \notin X$ and, by [28, Corollary 11.7.2], cl cone $C_{S} \subset X$. Consequently, $\left(\begin{array}{c}0_{n} \\ 1\end{array}\right) \notin$ cl cone $C_{S}$ and (iii) holds.

(iii) $\Rightarrow$ (i) Now assume that $0_{n+1} \notin$ eco $C_{S}$ and $\left(\begin{array}{c}0_{n} \\ 1\end{array}\right) \notin$ cl cone $C_{S}$.

First condition implies the existence of a vector $\left(\begin{array}{l}a \\ \alpha\end{array}\right) \in \mathbb{R}^{n+1}$ such that $\left(\begin{array}{l}a \\ \alpha\end{array}\right)^{\prime} z>0$ for all $z \in C_{S}$. Then

$$
a_{t}^{\prime} a+b_{t} \alpha>0
$$

for all $t \in S$.

By Proposition 1.1, second condition is equivalent to the consistency of the system $\left\{a_{t}^{\prime} x \geq b_{t}, t \in S\right\}$. Let $\bar{x}$ be a solution of such system.

It can be easily realized, from (3.1), that $x \in \mathbb{R}^{n}$, defined as

$$
x:= \begin{cases}-\frac{a}{\alpha}, & \text { if } \quad \alpha<0, \\ a+\bar{x}, & \text { if } \quad \alpha=0, \\ 2 \bar{x}+\frac{a}{\alpha}, & \text { if } \quad \alpha>0,\end{cases}
$$

is a solution of $\left\{a_{t}^{\prime} x>b_{t}, t \in S\right\}$.

Theorem 3.1. The following conditions are equivalent to each other:

(i) $\sigma=\left\{a_{t}^{\prime} x>b_{t}, t \in S ; a_{t}^{\prime} x \geq b_{t}, t \in W ; a_{t}^{\prime} x=b_{t}, t \in E\right\}$, with $S \neq \emptyset$, is consistent;

(ii) $0_{n+1} \notin$ eco $\left[\left\{\left(\begin{array}{c}a_{t} \\ b_{t}\end{array}\right), t \in S\right\}+\mathbb{R}_{+}\left\{\left(\begin{array}{c}a_{t} \\ b_{t}\end{array}\right), t \in W\right\}+\mathbb{R}\left\{\left(\begin{array}{c}a_{t} \\ b_{t}\end{array}\right), t \in E\right\} ;\left(\begin{array}{c}0_{n} \\ -1\end{array}\right)\right]$;

(iii) $0_{n+1} \notin$ eco $\left[\left\{\left(\begin{array}{c}a_{t} \\ b_{t}\end{array}\right), t \in S\right\}+\mathbb{R}_{+}\left\{\left(\begin{array}{c}a_{t} \\ b_{t}\end{array}\right), t \in W\right\}+\mathbb{R}\left\{\left(\begin{array}{c}a_{t} \\ b_{t}\end{array}\right), t \in E\right\}\right]$ and

$$
\left(\begin{array}{c}
0_{n} \\
1
\end{array}\right) \notin \operatorname{clcone}\left\{\left(\begin{array}{c}
a_{t} \\
b_{t}
\end{array}\right), t \in V ; \pm\left(\begin{array}{c}
a_{t} \\
b_{t}
\end{array}\right), t \in E\right\} \text {. }
$$

Proof. We assume $W \neq \emptyset \neq E$ (otherwise the proof is simpler). The proof is a straightforward consequence of Lemma 3.1 due to the equivalence between the consistency of $\sigma$ and the consistency of the system

$$
\sigma_{1}=\left\{\left(a_{s}+\alpha a_{w}+\beta a_{e}\right)^{\prime} x>b_{s}+\alpha b_{w}+\beta b_{e}, \quad(s, w, e, \alpha, \beta) \in S \times W \times E \times \mathbb{R}_{+} \times \mathbb{R}\right\}
$$

Since any solution of $\sigma$ is trivially a solution of $\sigma_{1}$, we consider a solution of $\sigma_{1}$, say $\bar{x}$.

Given $s \in S$, and taking arbitrarily $w \in W$ and $e \in E$, we have, for the index 
$(s, w, e, 0,0) \in S \times W \times E \times \mathbb{R}_{+} \times \mathbb{R}$, the strict inequality

$$
a_{s}^{\prime} \bar{x}>b_{s}
$$

Similarly, given $w \in W$, taking arbitrarily $s \in S, e \in E$ and $r>0$, we have, for the index $(s, w, e, r, 0) \in S \times W \times E \times \mathbb{R}_{+} \times \mathbb{R}$, the inequality

$$
\left(a_{s}+r a_{w}\right)^{\prime} \bar{x}>b_{s}+r b_{w} .
$$

Multiplying by $r^{-1}$ both sides of (3.3) and taking limits as $r \rightarrow+\infty$, we get

$$
a_{w}^{\prime} \bar{x} \geq b_{w}
$$

Finally, given $e \in E$, for any $s \in S, w \in W$ and $r \in \mathbb{R} \backslash\{0\}$, we have, for the index $(s, w, e, 0, r) \in S \times W \times E \times \mathbb{R}_{+} \times \mathbb{R}$, the inequality

$$
\left(a_{s}+r a_{e}\right)^{\prime} \bar{x}>b_{s}+r b_{e} .
$$

Multiplying by $|r|^{-1}$ both sides of (3.5) and taking limits as $r \rightarrow \pm \infty$, we get now

$$
a_{e}^{\prime} \bar{x}=b_{e}
$$

Therefore, from (3.2), (3.4) and (3.6), we conclude that $\bar{x}$ is a solution of $\sigma$.

Obviously, conditions (ii) and (iii) are equivalent to assert the consistency of associated systems in $\mathbb{R}^{n+1}$ (in the same vein, the alternative theorems establish the equivalence between the consistency of a given system and the inconsistency of an associated one). Observe that Proposition 2.1 provides a geometric and analytic description of the condition $0_{n+1} \notin$ eco $X$. Now we shall obtain some straightforward consequences of Theorem 3.1 (other consequences are obtained in [10] as corollaries of the next result).

Corollary 3.1 ([10, Proposition 2.1]). Let $\sigma$ be as in Theorem 3.1, with $E=\emptyset$. Then the following statements hold:

(i) If $\sigma$ is consistent, then

and

$$
\left(\begin{array}{c}
0_{n} \\
1
\end{array}\right) \notin \operatorname{clcone}\left\{\left(\begin{array}{c}
a_{t} \\
b_{t}
\end{array}\right), t \in T\right\}
$$

$$
0_{n+1} \notin \operatorname{conv}\left\{\left(\begin{array}{l}
a_{t} \\
b_{t}
\end{array}\right), t \in S\right\}+\operatorname{cone}\left\{\left(\begin{array}{l}
a_{t} \\
b_{t}
\end{array}\right), t \in W\right\}
$$


(ii) If (3.7) and (3.8) hold and the set in (3.8) is closed, then $\sigma$ is consistent.

Proof. (i) According to Lemma 2.1, part (i), we have

$$
\operatorname{conv} C_{S}+\operatorname{cone} C_{W}=\operatorname{conv}\left[C_{S}+\mathbb{R}_{+} C_{W}\right] \subset \operatorname{eco}\left[C_{S}+\mathbb{R}_{+} C_{W}\right]
$$

and (3.7) and (3.8) hold by straightforward application of Theorem 3.1.

(ii) We can reformulate (3.8) as

$$
0_{n+1} \notin \operatorname{conv}\left[C_{S}+\mathbb{R}_{+} C_{W}\right]
$$

Since we are assuming the closedness of the convex set in (3.10), it is equal to the set eco $\left[C_{S}+\mathbb{R}_{+} C_{W}\right]$. Then Theorem 3.1 applies again.

The following example illustrates the dubious case in Corollary 3.1.

Example 3.1. Let $\sigma=\left\{t x_{1}+x_{2}>-t^{2}, t \in[-1,1] \backslash\{0\} ;-x_{2}>0\right\}$.

Obviously, $0_{2}$ is a solution of the relaxed system of $\sigma$,

$$
\bar{\sigma}=\left\{t x_{1}+x_{2} \geq-t^{2}, t \in[-1,1] \backslash\{0\} ;-x_{2} \geq 0\right\}
$$

and, by Proposition 1.1, (3.7) holds. On the other hand, the set in (3.8) is here

$$
\begin{gathered}
Y=\operatorname{conv}\left\{\left(\begin{array}{c}
t \\
1 \\
-t^{2}
\end{array}\right), t \in[-1,1] \backslash\{0\} ;\left(\begin{array}{c}
0 \\
-1 \\
0
\end{array}\right)\right\} \\
\left.\left.=\operatorname{conv}\left\{\left(\begin{array}{c}
t \\
1 \\
-t^{2}
\end{array}\right), t \in[-1,1] ;\left(\begin{array}{c}
0 \\
-1 \\
0
\end{array}\right)\right\} \backslash\right]\left(\begin{array}{c}
0 \\
-1 \\
0
\end{array}\right),\left(\begin{array}{l}
0 \\
1 \\
0
\end{array}\right)\right],
\end{gathered}
$$

which is not closed and does not contain $0_{3}$ (see Figure 3.1). Hence, (3.7) and (3.8) hold, but we cannot apply Corollary 3.1 .

On the other hand, $Y$ is not evenly convex because $0_{3} \in(\operatorname{cl} Y) \backslash Y$ but for any hyperplane $H$ such that $0_{3} \in H$, we have $H \cap Y \neq \emptyset$. In fact,

$$
\operatorname{eco}\left\{\left(\begin{array}{c}
t \\
1 \\
-t^{2}
\end{array}\right), t \in[-1,1] \backslash\{0\} ;\left(\begin{array}{c}
0 \\
-1 \\
0
\end{array}\right)\right\}=\operatorname{cl} Y
$$

and, since the first condition in Theorem 3.1(iii) fails, $\sigma$ turns out to be inconsistent. 


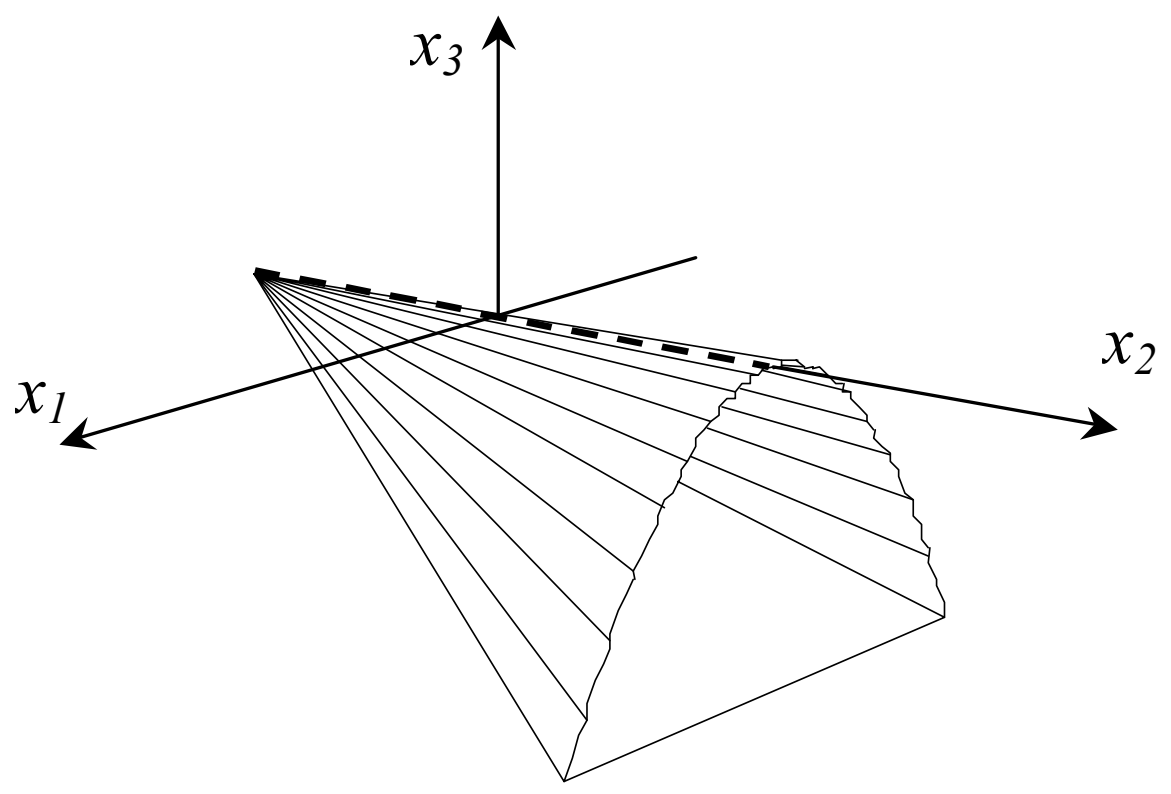

Figure 3.1

Corollary 3.2. Let $\sigma$ be as in Theorem 3.1, with $E=\emptyset$. Assume that $\left\{a_{t}^{\prime} x \geq b_{t}, t \in W\right\}$ is consistent and that cone $\left\{\left(\begin{array}{c}a_{t} \\ b_{t}\end{array}\right), t \in T\right\}$ and conv $\left\{\left(\begin{array}{c}a_{t} \\ b_{t}\end{array}\right), t \in S\right\}+\operatorname{cone}\left\{\left(\begin{array}{c}a_{t} \\ b_{t}\end{array}\right), t \in W\right\}$ are closed sets. Then one and only one of the following alternatives hold:

(i) $\sigma$ is consistent.

(ii) There exists $\lambda \in \mathbb{R}_{+}^{(T)}$ such that at least one of the numbers $\lambda_{t}, t \in S$, is nonzero, and

$$
\sum_{t \in T} \lambda_{t} a_{t}=0_{n} \quad \text { and } \quad \sum_{t \in T} \lambda_{t} b_{t} \geq 0
$$

Proof. We shall prove the equivalence between (ii) and the negation of (i). According to Corollary 3.1, (i) fails if and only if either

$$
\left(\begin{array}{c}
0_{n} \\
1
\end{array}\right) \in \operatorname{cone} C_{T}
$$

or

$$
0_{n+1} \in \operatorname{conv} C_{S}+\text { cone } C_{W}
$$

If (3.12) holds, we can write

$$
\left(\begin{array}{c}
0_{n} \\
1
\end{array}\right)=\sum_{t \in T} \lambda_{t}\left(\begin{array}{c}
a_{t} \\
b_{t}
\end{array}\right), \lambda \in \mathbb{R}_{+}^{(T)},
$$

so that (3.11) holds. Moreover, there exists $t \in S$ such that $\lambda_{t}>0$ (otherwise $\left(\begin{array}{c}0_{n} \\ 1\end{array}\right) \in \operatorname{cone} C_{W}$, and this is impossible due to the consistency of $\left\{a_{t}^{\prime} x \geq b_{t}, t \in W\right\}$ ). 
Alternatively, if (3.13) holds, there exists $\lambda \in \mathbb{R}_{+}^{(T)}$ such that

$$
0_{n+1}=\sum_{t \in T} \lambda_{t}\left(\begin{array}{l}
a_{t} \\
b_{t}
\end{array}\right) \quad \text { and } \quad \sum_{t \in S} \lambda_{t}=1,
$$

and (3.11) holds again with $\lambda_{t}>0$ for at least one index $t \in S$.

Consequently, (ii) holds in both cases. and

Now we assume that (ii) holds, i.e., there exist $\lambda \in \mathbb{R}_{+}^{(T)}$ and $\alpha \geq 0$ such that $\sum_{t \in S} \lambda_{t}>0$

$$
\left(\begin{array}{c}
0_{n} \\
\alpha
\end{array}\right)=\sum_{t \in T} \lambda_{t}\left(\begin{array}{l}
a_{t} \\
b_{t}
\end{array}\right)
$$

If $\alpha>0$, dividing both members of (3.14) by $\alpha$, we get

$$
\left(\begin{array}{c}
0_{n} \\
1
\end{array}\right)=\sum_{t \in T}\left(\alpha^{-1} \lambda_{t}\right)\left(\begin{array}{c}
a_{t} \\
b_{t}
\end{array}\right) \in \operatorname{cone} C_{T}
$$

and $\sigma$ is inconsistent.

Alternatively, if $\alpha=0$, dividing both members of (3.14) by $\mu:=\sum_{t \in S} \lambda_{t}$, we get

$$
0_{n+1}=\sum_{t \in S}\left(\mu^{-1} \lambda_{t}\right)\left(\begin{array}{l}
a_{t} \\
b_{t}
\end{array}\right)+\sum_{t \in W}\left(\mu^{-1} \lambda_{t}\right)\left(\begin{array}{l}
a_{t} \\
b_{t}
\end{array}\right) \in \operatorname{conv} C_{S}+\operatorname{cone} C_{W}
$$

so that $\sigma$ is inconsistent by Corollary 3.1.

Hence, (i) fails in both cases.

If $S$ is compact and cone $\left\{\left(\begin{array}{c}a_{t} \\ b_{t}\end{array}\right), t \in W\right\}$ is closed, then the closedness assumptions in Corollary 3.2 hold. In particular, if $S$ and $W$ are finite, then Corollary 3.2 becomes [ 28, Theorem 22.2].

The next result generalizes the Extended Motzkin's Alternative Theorem (see [ 11, p. 68]).

Corollary 3.3. An homogeneous system

$$
\sigma=\left\{a_{t}^{\prime} x>0, t \in S ; a_{t}^{\prime} x \geq 0, t \in W ; a_{t}^{\prime} x=0, t \in E\right\}
$$

is consistent if and only if

$$
0_{n} \notin \operatorname{eco}\left[\left\{a_{t}, t \in S\right\}+\mathbb{R}_{+}\left\{a_{t}, t \in W\right\}+\mathbb{R}\left\{a_{t}, t \in E\right\}\right]
$$


Proof. Combining Theorem 3.1 and Proposition 2.3, $\sigma$ is consistent if and only if

$$
\begin{aligned}
0_{n+1} \notin \text { eco }\left[\left\{\left(\begin{array}{c}
a_{t} \\
0
\end{array}\right), t \in S\right\}+\mathbb{R}_{+}\left\{\left(\begin{array}{c}
a_{t} \\
0
\end{array}\right), t \in W\right\}+\mathbb{R}\left\{\left(\begin{array}{c}
a_{t} \\
0
\end{array}\right), t \in E\right\}\right] \\
=\operatorname{eco}\left[\left\{a_{t}, t \in S\right\}+\mathbb{R}_{+}\left\{a_{t}, t \in W\right\}+\mathbb{R}\left\{a_{t}, t \in E\right\}\right] \times\{0\}
\end{aligned}
$$

if and only if (3.15) holds.

\section{Consequence relations}

A linear relation $a^{\prime} x \geq b\left(a^{\prime} x>b\right.$ or $\left.a^{\prime} x=b\right)$ is a consequence of a system $\sigma$ if $a^{\prime} \bar{x} \geq b$ $\left(a^{\prime} \bar{x}>b\right.$ or $a^{\prime} \bar{x}=b$ ) holds for every $\bar{x} \in \mathbb{R}^{n}$ solution of $\sigma$. If $\sigma$ is inconsistent, then any linear inequality (or equality) is a consequence of $\sigma$. So we assume throughout this section that

$$
\sigma=\left\{a_{t}^{\prime} x>b_{t}, t \in S ; a_{t}^{\prime} x \geq b_{t}, t \in W ; a_{t}^{\prime} x=b_{t}, t \in E\right\}
$$

is consistent. We denote $\bar{\sigma}=\left\{a_{t}^{\prime} x \geq b_{t}, t \in V ; a_{t}^{\prime} x=b_{t}, t \in E\right\}$.

Proposition 4.1. A weak inequality $a^{\prime} x \geq b$ is a consequence of $\sigma$ if and only if

$$
\left(\begin{array}{l}
a \\
b
\end{array}\right) \in \operatorname{clcone}\left\{\left(\begin{array}{l}
a_{t} \\
b_{t}
\end{array}\right), t \in V ; \pm\left(\begin{array}{l}
a_{t} \\
b_{t}
\end{array}\right), t \in E ;\left(\begin{array}{c}
0_{n} \\
-1
\end{array}\right)\right\} .
$$

Proof. If $a^{\prime} x \geq b$ is a consequence of $\sigma$, then it is also a consequence of $\bar{\sigma}$. In fact, let $\bar{x}$ be an arbitrary solution of $\bar{\sigma}$ and take an arbitrary $x^{1}$ solution of $\sigma$. Then, for every $\left.\lambda \in\right] 0,1[$, we get a solution of $\sigma$ of the form $x(\lambda):=(1-\lambda) \bar{x}+\lambda x^{1}$. Since $a^{\prime} x \geq b$ is a consequence of $\sigma$, we get $a^{\prime} x(\lambda) \geq b$ and taking limits as $\lambda \rightarrow 0^{+}$we obtain $a^{\prime} \bar{x} \geq b$. Since $a^{\prime} x \geq b$ is a consequence of a consistent system $\bar{\sigma}=\left\{a_{t}^{\prime} x \geq b_{t}, t \in V ; a_{t}^{\prime} x=b_{t}, t \in E\right\}$, we can apply Proposition 1.2 to get (4.1).

Corollary 4.1. An equation $a^{\prime} x=b$ is a consequence of $\sigma$ if and only if

$$
\pm\left(\begin{array}{l}
a \\
b
\end{array}\right) \in \operatorname{clcone}\left\{\left(\begin{array}{l}
a_{t} \\
b_{t}
\end{array}\right), t \in V ; \pm\left(\begin{array}{l}
a_{t} \\
b_{t}
\end{array}\right), t \in E ;\left(\begin{array}{c}
0_{n} \\
-1
\end{array}\right)\right\} .
$$

We have shown that $a^{\prime} x \geq b\left(a^{\prime} x=b\right)$ is a consequence of $\sigma$ if and only if it is a consequence of $\bar{\sigma}$. So, this is a necessary condition for $a^{\prime} x>b$ to be a consequence of $\sigma$.

Inspired by [18], we say that a strict inequality $a^{\prime} x>b$ is a legal linear combination of $\sigma$ if 
there exists $\lambda \in \mathbb{R}_{+}^{(V)}$ such that $\lambda_{t}>0$ for at least one index $t \in S$, and $\mu \in \mathbb{R}^{(E)}$ such that

$$
\left(\begin{array}{l}
a \\
b
\end{array}\right)=\sum_{t \in V} \lambda_{t}\left(\begin{array}{l}
a_{t} \\
b_{t}
\end{array}\right)+\sum_{t \in E} \mu_{t}\left(\begin{array}{l}
a_{t} \\
b_{t}
\end{array}\right)
$$

Proposition 4.2. A strict inequality $a^{\prime} x>b$ is a consequence of $\sigma$ if and only if at least one of the following conditions hold:

(i) $\left(\begin{array}{c}0_{n} \\ 1\end{array}\right) \in \operatorname{cl}$ cone $\left\{\left(\begin{array}{c}a_{t} \\ b_{t}\end{array}\right), t \in V ; \pm\left(\begin{array}{c}a_{t} \\ b_{t}\end{array}\right), t \in E ;-\left(\begin{array}{c}a \\ b\end{array}\right)\right\}$.

(ii) $0_{n+1} \in$ eco $\left[\left\{\left(\begin{array}{l}a_{t} \\ b_{t}\end{array}\right), t \in S\right\}+\mathbb{R}_{+}\left\{\left(\begin{array}{l}a_{t} \\ b_{t}\end{array}\right), t \in W ;-\left(\begin{array}{l}a \\ b\end{array}\right)\right\}+\mathbb{R}\left\{\left(\begin{array}{l}a_{t} \\ b_{t}\end{array}\right), t \in E\right\}\right]$.

If $a^{\prime} x>b$ is a legal linear combination of $\bar{\sigma} \cup\left\{0_{n}^{\prime} x>-1\right\}$, then (i) holds. The converse is true if

is closed.

$$
\text { cone }\left\{\left(\begin{array}{l}
a_{t} \\
b_{t}
\end{array}\right), t \in V ; \pm\left(\begin{array}{l}
a_{t} \\
b_{t}
\end{array}\right), t \in E ;-\left(\begin{array}{l}
a \\
b
\end{array}\right)\right\}
$$

If $a^{\prime} x>b$ is a legal linear combination of $\sigma$, then (ii) holds. The converse is true if

$$
\operatorname{conv}\left[\left\{\left(\begin{array}{l}
a_{t} \\
b_{t}
\end{array}\right), t \in S\right\}+\mathbb{R}_{+}\left\{\left(\begin{array}{l}
a_{t} \\
b_{t}
\end{array}\right), t \in W ;-\left(\begin{array}{l}
a \\
b
\end{array}\right)\right\}+\mathbb{R}\left\{\left(\begin{array}{l}
a_{t} \\
b_{t}
\end{array}\right), t \in E\right\}\right]
$$

is evenly convex.

Proof. A strict inequality $a^{\prime} x>b$ is a consequence of $\sigma$ if and only if

$$
\sigma \cup\left\{a^{\prime} x \leq b\right\}=\left\{a_{t}^{\prime} x>b_{t}, t \in S ; a_{t}^{\prime} x \geq b_{t}, t \in W ; a_{t}^{\prime} x=b_{t}, t \in E ;-a^{\prime} x \geq-b\right\}
$$

is inconsistent. Recalling Theorem 3.1, this is equivalent to assert that either (i) or (ii) holds.

Now we assume that $a^{\prime} x>b$ is a legal linear combination of $\bar{\sigma} \cup\left\{0_{n}^{\prime} x>-1\right\}$. Then we can write

$$
\left(\begin{array}{l}
a \\
b
\end{array}\right)=\sum_{t \in V} \lambda_{t}\left(\begin{array}{l}
a_{t} \\
b_{t}
\end{array}\right)+\sum_{t \in E} \mu_{t}\left(\begin{array}{c}
a_{t} \\
b_{t}
\end{array}\right)+\gamma\left(\begin{array}{c}
0_{n} \\
-1
\end{array}\right), \lambda \in \mathbb{R}_{+}^{(V)}, \mu \in \mathbb{R}^{(E)}, \gamma>0
$$

Then we have

$$
\left(\begin{array}{c}
0_{n} \\
1
\end{array}\right)=\sum_{t \in V}\left(\gamma^{-1} \lambda_{t}\right)\left(\begin{array}{l}
a_{t} \\
b_{t}
\end{array}\right)+\sum_{t \in E}\left(\gamma^{-1} \mu_{t}\right)\left(\begin{array}{l}
a_{t} \\
b_{t}
\end{array}\right)-\gamma^{-1}\left(\begin{array}{l}
a \\
b
\end{array}\right) \in \text { cone }\left[C_{V} \cup\left( \pm C_{E}\right) \cup\left\{-\left(\begin{array}{l}
a \\
b
\end{array}\right)\right\}\right]
$$


and (i) holds. Conversely, if (i) holds and the convex cone in (4.2) is closed, we can write

$$
\left(\begin{array}{c}
0_{n} \\
1
\end{array}\right)=\sum_{t \in V} \lambda_{t}\left(\begin{array}{c}
a_{t} \\
b_{t}
\end{array}\right)+\sum_{t \in E} \mu_{t}\left(\begin{array}{c}
a_{t} \\
b_{t}
\end{array}\right)-\gamma\left(\begin{array}{l}
a \\
b
\end{array}\right), \lambda \in \mathbb{R}_{+}^{(V)}, \mu \in \mathbb{R}^{(E)}, \gamma \geq 0 .
$$

If $\gamma=0,\left(\begin{array}{c}0_{n} \\ 1\end{array}\right) \in$ cone $\left[C_{V} \cup\left( \pm C_{E}\right)\right]$, but this entails the inconsistency of $\bar{\sigma}$.

Thus $\gamma>0$ and, from (4.4), we get

$$
\left(\begin{array}{l}
a \\
b
\end{array}\right)=\sum_{t \in V}\left(\gamma^{-1} \lambda_{t}\right)\left(\begin{array}{l}
a_{t} \\
b_{t}
\end{array}\right)+\sum_{t \in E}\left(\gamma^{-1} \mu_{t}\right)\left(\begin{array}{l}
a_{t} \\
b_{t}
\end{array}\right)+\gamma^{-1}\left(\begin{array}{c}
0_{n} \\
-1
\end{array}\right)
$$

so that $a^{\prime} x>b$ is a legal linear combination of $\bar{\sigma} \cup\left\{0_{n}^{\prime} x>-1\right\}$.

If $a^{\prime} x>b$ is a legal linear combination of $\sigma$, then there exist $\lambda \in \mathbb{R}_{+}^{(V)}$ and $\mu \in \mathbb{R}^{(E)}$ such that $\gamma:=\sum_{t \in S} \lambda_{t}>0$ and

$$
\left(\begin{array}{l}
a \\
b
\end{array}\right)=\sum_{t \in V} \lambda_{t}\left(\begin{array}{l}
a_{t} \\
b_{t}
\end{array}\right)+\sum_{t \in E} \mu_{t}\left(\begin{array}{l}
a_{t} \\
b_{t}
\end{array}\right)
$$

By Proposition 2.2, we have

$$
\begin{gathered}
0_{n+1}=\sum_{t \in S}\left(\gamma^{-1} \lambda_{t}\right)\left(\begin{array}{l}
a_{t} \\
b_{t}
\end{array}\right)+\sum_{t \in W}\left(\gamma^{-1} \lambda_{t}\right)\left(\begin{array}{l}
a_{t} \\
b_{t}
\end{array}\right)+\sum_{t \in E}\left(\gamma^{-1} \mu_{t}\right)\left(\begin{array}{l}
a_{t} \\
b_{t}
\end{array}\right)-\gamma^{-1}\left(\begin{array}{l}
a \\
b
\end{array}\right) \\
\in \operatorname{conv} C_{S}+\operatorname{cone}\left[C_{W} \cup\left\{-\left(\begin{array}{l}
a \\
b
\end{array}\right)\right\}\right]+\operatorname{span} C_{E} \\
\subset \operatorname{eco}\left[\operatorname{conv} C_{S}+\operatorname{cone}\left[C_{W} \cup\left\{-\left(\begin{array}{l}
a \\
b
\end{array}\right)\right\}\right]+\operatorname{span} C_{E}\right] \\
=\operatorname{eco}\left[C_{S}+\mathbb{R}_{+}\left(C_{W} \cup\left\{-\left(\begin{array}{l}
a \\
b
\end{array}\right)\right\}\right)+\mathbb{R} C_{E}\right],
\end{gathered}
$$

i.e. (ii) holds.

Finally, we assume that (ii) holds and the set in (4.3) is evenly convex. Then, by Lemma 2.1,

$$
0_{n+1} \in \operatorname{conv} C_{S}+\text { cone }\left[C_{W} \cup\left\{-\left(\begin{array}{l}
a \\
b
\end{array}\right)\right\}\right]+\operatorname{span} C_{E}
$$

and there exist $\lambda \in \mathbb{R}_{+}^{(V)}, \mu \in \mathbb{R}^{(E)}$ and $\gamma \geq 0$ such that $\sum_{t \in S} \lambda_{t}=1$ and

$$
\gamma\left(\begin{array}{l}
a \\
b
\end{array}\right)=\sum_{t \in V} \lambda_{t}\left(\begin{array}{l}
a_{t} \\
b_{t}
\end{array}\right)+\sum_{t \in E} \mu_{t}\left(\begin{array}{l}
a_{t} \\
b_{t}
\end{array}\right)
$$

If $\gamma=0$, then (4.5) yields

$0_{n+1} \in \operatorname{conv} C_{S}+$ cone $C_{W}+\operatorname{span} C_{E}=\operatorname{conv}\left[C_{S}+\mathbb{R}_{+} C_{W}+\mathbb{R} C_{E}\right] \subset \operatorname{eco}\left[C_{S}+\mathbb{R}_{+} C_{W}+\mathbb{R} C_{E}\right]$ 
and $\sigma$ would be inconsistent by Theorem 3.1, contradicting the assumption. Hence $\gamma>0$ and (4.5) entails

$$
\left(\begin{array}{l}
a \\
b
\end{array}\right)=\sum_{t \in V}\left(\gamma^{-1} \lambda_{t}\right)\left(\begin{array}{l}
a_{t} \\
b_{t}
\end{array}\right)+\sum_{t \in E}\left(\gamma^{-1} \mu_{t}\right)\left(\begin{array}{l}
a_{t} \\
b_{t}
\end{array}\right)
$$

with $\sum_{t \in S} \gamma^{-1} \lambda_{t}=\gamma^{-1}>0$, so that $a^{\prime} x>b$ is a legal linear combination of $\sigma$.

If $S, W$ and $E$ are finite, then the convex cone in (4.2) is polyhedral and the set in (4.3), which can be written as

$$
\operatorname{conv}\left\{\left(\begin{array}{l}
a_{t} \\
b_{t}
\end{array}\right), t \in S\right\}+\operatorname{cone}\left\{\left(\begin{array}{l}
a_{t} \\
b_{t}
\end{array}\right), t \in W ;-\left(\begin{array}{l}
a \\
b
\end{array}\right)\right\}+\operatorname{span}\left\{\left(\begin{array}{l}
a_{t} \\
b_{t}
\end{array}\right), t \in E\right\}
$$

(by Lemma 2.1, part (ii)), is actually a polyhedral convex set.

So, $a^{\prime} x>b$ is a consequence of a consistent finite system $\sigma$ if and only if $a^{\prime} x>b$ is a legal linear combination of either $\sigma$ or $\bar{\sigma} \cup\left\{0_{n}^{\prime} x>-1\right\}$. This result, for $E=\emptyset$, is a reformulation of [18, Theorem II], and it is not valid for semi-infinite systems (unless the two additional assumptions in Proposition 4.2 hold), as the next example shows.

Example 4.1. The inequality $x_{2}>0$, in $\mathbb{R}^{2}$, is a consequence of

$$
\sigma=\left\{-2 t x_{1}+x_{2}>-t^{2}, t \in U ;-x_{1}+x_{2}>0\right\}
$$

where $U=]-1,0[$, as far as the solution set of $\sigma$ is the interior of the epigraph of the function $f: \mathbb{R} \rightarrow \mathbb{R}$, defined as

$$
f\left(x_{1}\right)= \begin{cases}-2 x_{1}-1, & x_{1}<-1 \\ x_{1}^{2}, & -1 \leq x_{1} \leq 0 \\ x_{1}, & x_{1}>0 .\end{cases}
$$

Nevertheless, $x_{2}>0$ fails to be legal linear combination of $\bar{\sigma} \cup\left\{0_{2}^{\prime} x>-1\right\}$ or $\sigma$, since the following systems are inconsistent:

$$
\left(\begin{array}{l}
0 \\
1 \\
0
\end{array}\right)=\sum_{t \in U} \lambda_{t}\left(\begin{array}{c}
-2 t \\
1 \\
-t^{2}
\end{array}\right)+\gamma\left(\begin{array}{c}
-1 \\
1 \\
0
\end{array}\right)+\mu\left(\begin{array}{c}
0 \\
0 \\
-1
\end{array}\right), \quad \lambda \in \mathbb{R}_{+}^{(U)}, \gamma \geq 0, \mu>0
$$

and

$$
\left(\begin{array}{l}
0 \\
1 \\
0
\end{array}\right)=\sum_{t \in U} \lambda_{t}\left(\begin{array}{c}
-2 t \\
1 \\
-t^{2}
\end{array}\right)+\gamma\left(\begin{array}{c}
-1 \\
1 \\
0
\end{array}\right), \quad \lambda \in \mathbb{R}_{+}^{(U)}, \gamma \geq 0, \sum_{t \in U} \lambda_{t}+\gamma>0
$$


Actually statement (ii) in Proposition 4.2 holds but the set in (4.3) is not evenly convex (see Figure 4.1).

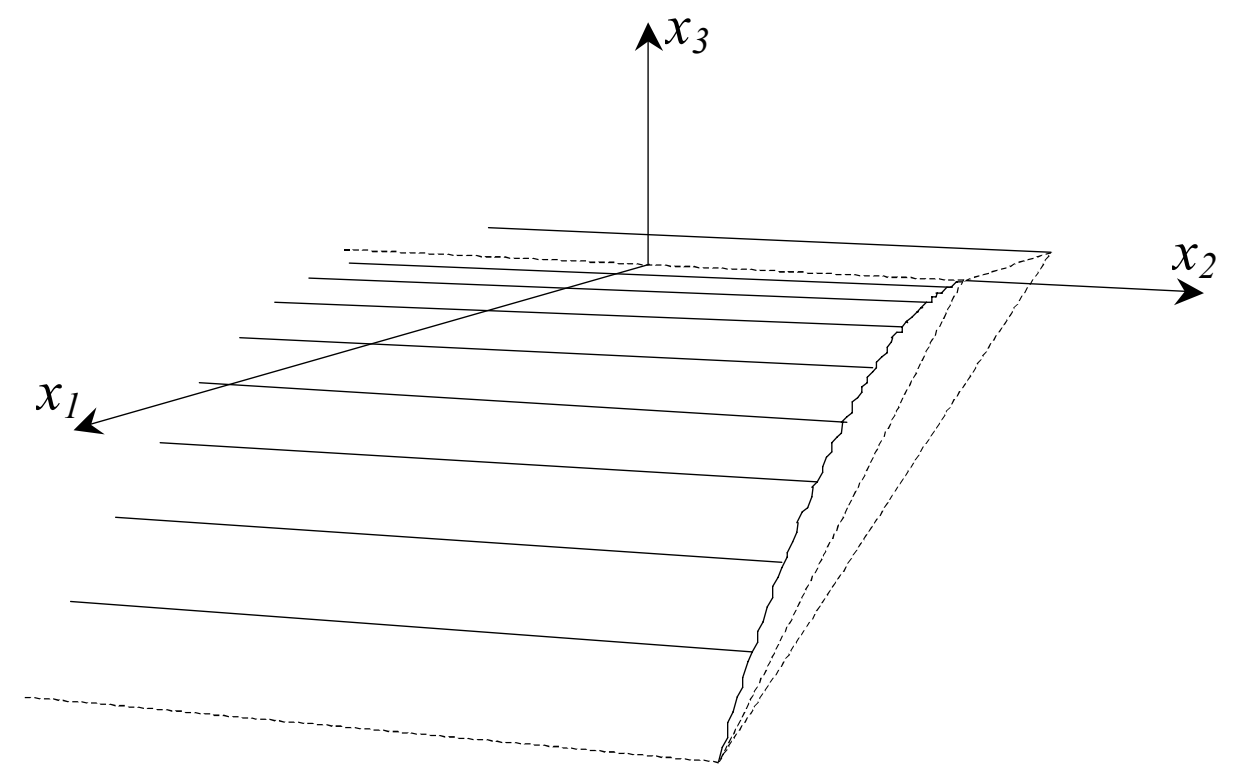

Figure 4.1

\section{REFERENCES}

[1] R. Bellman and K. Fan, On systems of linear inequalities in hermitian matrix variables. In: V. L. Klee (ed.), Convexity. Proceedings of Symposia in Pure Mathematics VII, Providence, RI: American Mathematical Society, (1963) 1-11.

[2] P. S. Bradley, U. M. Fayyad and O. L. Mangasarian, Data mining: Overview and optimization opportunities, INFORMS Journal on Computing 11 (1999) 217-238.

[3] C. C. Braunschweiger, An extension of the nonhomogeneous Farkas theorem, American Mathematical Monthly 69 (1962) 969-975.

[4] W. B. Carver, Systems of linear inequalities, Annals of Mathematics 23 (1921-22) 212-220.

[5] A. Daniilidis and J.-E. Martínez-Legaz, Characterizations of evenly convex sets and evenly quasiconvex functions, Journal of Mathematical Analysis and Applications 273 (2002) 58-66.

[6] K. Fan, On infinite systems of linear inequalities, Journal of Mathematical Analysis and Applications 21 (1968) 475-478.

[7] W. Fenchel, A remark on convex sets and polarity, Communications du Séminaire Mathéma- 
tique de l'Université de Lund, Supplement (1952) 82-89.

[8] D. Gale, The theory of linear economic models, McGraw-Hill Book Company, New York, 1960.

[9] M. A. Goberna, V. Jornet and R. Puente, Optimización lineal. Teoría, métodos y modelos, McGraw-Hill, Madrid, Spain, 2004.

[10] M. A. Goberna, V. Jornet and M. M. L. Rodríguez, On linear systems containing strict inequalities, Linear Algebra and its Applications 360 (2003) 151-171.

[11] M. A. Goberna and M. A. López, Linear semi-infinite optimization, J. Wiley, Chichester, England, 1998.

[12] M. A. Goberna, M. A. López, J. T. Pastor and E. Vercher, Alternative theorems for infinite systems with applications to semi-infinite games, Nieuw Archief voor Wiskunde IV, Ser. 2 (1984) 218-234.

[13] P. Gordan, Über die Auflösungen linearer Gleichungen mit reelen Coefficienten, Mathematische Annalen 6 (1873) 23-28.

[14] A. Haar, Über lineare ungleichungen, Acta Math. Szeged 2 (1924) 1-14.

[15] V. Jeyakumar, Farkas' Lemma: Generalizations. In: Encyclopedia of Optimization, Vol. 2 (2001) 87-91, Kluwer Academic Publishers, Dordrecht, The Netherlands.

[16] V. Jeyakumar, Characterizing set containments involving infinite convex constraints and reverseconvex constraints, SIAM Journal on Optimization. 13 (2003) 947-959.

[17] V. Jeyakumar and B. Waterhouse, Data classification via separable convex programming, Appl. Math. Research Report AMR00/13, University of New South Wales, Sidney, Australia, 2000.

[18] H. W. Kuhn, Solvability and consistency for linear equations and inequalities, American Mathematical Monthly 63 (1956) 217-232.

[19] O. L. Mangasarian, Nonlinear programming, SIAM's Classics in Applied Mathematics, Philadelphia, USA, 1994.

[20] O. L. Mangasarian, Mathematical programming in data mining, Data Mining and Knowledge Discovery 1 (1997) 183-201.

[21] O. L. Mangasarian, Set containment characterization, Journal of Global Optimization 24 (2002) 473-480.

[22] J.-E. Martínez-Legaz, A generalized concept of conjugation, in: Optimization, Theory and Algorithms, edited by J.-B. Hiriart-Urruty, W. Oettli and J. Stoer, Lecture Notes in Pure and Ap- 
plied Mathematics 86 (1983) 45-59, Marcel Dekker, New York, NY.

[23] J.-E. Martínez-Legaz, Quasiconvex duality theory by generalized conjugation methods, Optimization 19 (1988) 603-652.

[24] J.-E. Martínez-Legaz, Duality between direct and indirect utility functions under minimal hypotheses, Journal of Mathematical Economics 20 (1991) 199-209.

[25] T. S. Motzkin, Beiträge zur Theorie der linearen Ungleichungen, Azriel, Jerusalem, 1936; transl. in Theodore S. Motzkin: Selected papers (D. Cantor, B. Gordon and B. Rothschild, eds.), Birkhäuser, Boston, 1983, pp. 1-80.

[26] U. Passy and E. Z. Prisman, Conjugacy in quasiconvex programming, Mathematical Programming 30 (1984) 121-146.

[27] J.-P. Penot and M. Volle, On quasiconvex duality, Mathematics of Operations Research 15 (1990) 597-625.

[28] R. T. Rockafellar, Convex Analysis, Princeton Univ. Press, Princeton, New Jersey, 1970.

[29] J. Stoer and C. Witzgall, Convexity and optimization in finite dimensions I, Springer-Verlag Berlin, Heidelberg, Germany, 1970.

[30] P. Szilágyi, Nonhomogeneous linear theorems of the alternative, Pure Mathematics and Applications 10 (1999) 141-159.

[31] Y. J. Zhu, Generalizations of some fundamental theorems on linear inequalities, Acta Mathematica Sinica 16 (1966) 25-39. 